\title{
Wood Veneer Defect Detection System Based on Machine Vision
}

\author{
Fan Yang ${ }^{a}{ }^{*}$, Yuzeng Wang ${ }^{b}$, Shibing Wang ${ }^{c}$ and Yunmei Cheng ${ }^{d}$ \\ School of Mechanical Engineering, University of Jinan, Jinan 250022, China \\ a, *yangfchn@foxmail.com, bme_wangyz@ujn.edu.cn, 'wang_shibing@qq.com, \\ d1713472489@qq.com
}

\begin{abstract}
In view of the problems of low surface detection efficiency and high error detection rate in domestic wood skin, a corresponding detection system is designed to identify four types of wood skin defects such as dead knot, slipknot, hole and crack on the surface of the wood. Based on the image recognition of industrial cameras, the detection system can collect data in real time and quickly. Then the system can recognize and classify the collected images. The system is of great significance to the rational utilization of wood. The experimental results show that the detection system is safe and reliable, and has high accuracy.
\end{abstract}

Keywords: Wood Veneer, Defect Detection System, Machine Vision.

\section{Introduction}

The wood-based panel industry is an important part of China's wood processing industry. In China's wood-based panel products, plywood started the earliest and the development speed is the fastest. Plywood has always been the leading product in the wood-based panel industry of our country. It is mainly used in furniture manufacturing, decoration, decoration, packaging and construction of [1]. Plywood is widely used in many fields, making the market a great demand for plywood, and the quality of the wood veneer, which is the primary raw material for plywood, determines the market value of plywood. There is a huge gap in the current wood veneer raw materials in China. Although the annual output of wood plywood in 2016 is close to 170 million cubic meters, the comprehensive utilization rate of the wood veneer is not high due to the surface defects of the wood veneer. Therefore, it is of great value and significance to classify the defects of the wood veneer.

At present, the detection of the surface defects of the wood veneer is basically the method of artificial detection. This method of identifying the surface defects is mainly based on human vision. The human eye is prone to fatigue in a long time work. The recognition rate will decrease gradually and the accuracy will be reduced, and the problem of error detection is easy to appear. At the same time, due to the different experience of different workers, the test results will not be exactly the same, which affects the stability of the surface detection quality of the veneer surface inspection [2-3]. With the intensive development of the wood processing industry and the demanding of the high quality of the wood surface processing quality, the traditional artificial detection method has been difficult to meet the processing and production of wood products [4]. In recent years, machine vision technology has been applied in a wide range of fields because of its advantages of non-contact, high automation and high efficiency in the field of industrial defect detection, and has achieved good application effect [5-8].

In view of the above problems, a wood veneer defect detection system is designed. The system is based on machine vision technology and analyzes and collects images collected. According to the detection and recognition of the image of the surface defect of the wood veneer, and the output of the detection results, the real-time detection and sorting of the defective wood is completed, which greatly improves the accuracy and efficiency of the detection. 


\section{Detection System Structure}

The principle of hardware composition of the on-line detection system designed in this paper is shown in Figure 1, which is mainly composed of industrial camera, light source, shading box, transmission mechanism, and fixed support structure and so on.

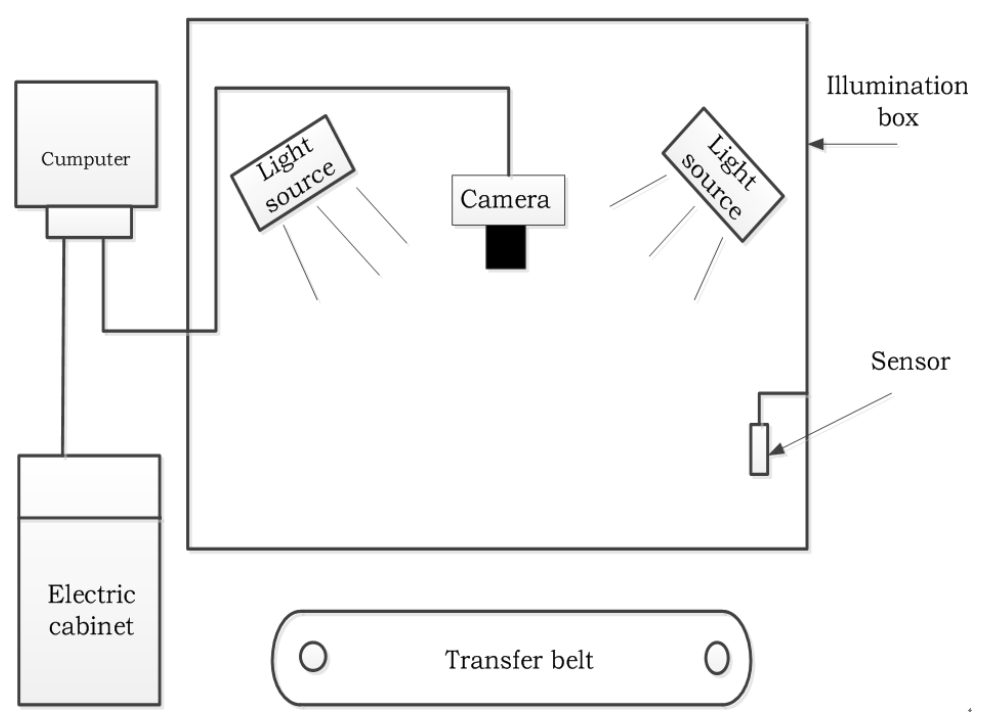

Fig. 1 Frame diagram of detection system.

The detection process is as follows: when the main control switch of the detection system is opened, the two sets of light sources are opened and the motor drives the conveyor belt to carry the movement of the wood veneer. When the photoelectric sensor installed on the fixed bracket detects the movement of the wood veneer on the conveyor belt, it will transmit the signal to the PLC. After detecting the input signal, PLC triggers the industrial camera to collect the surface image of the wood skin, and transmits the captured wooden skin image to the computer. Then the image features of the collected wood veneer are extracted and analyzed, and then the defect on the surface of the pending skin is judged. If there are defects on the surface of the veneer which do not meet the production requirements, PLC will control the sorting device and sort out the defective wood veneer.

\section{System Hardware Design}

\subsection{Camera Type Selection}

In order to make the subsequent software detection and recognition accurate and reliable, this requires that the images collected by the early stage camera have higher image quality. The camera selection of this detection system is shown in Figure 2. The camera is a mercury series industrial camera of Daheng Image Company. It can work steadily in a variety of harsh environments with high definition, high precision, and low noise and so on. It is compact, compact, durable and easy to install and use.

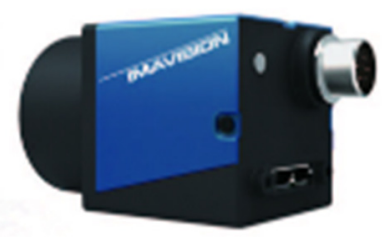

Fig. 2 Camera.

\subsection{Light Source Design}

Because the actual production system needs a long time to work, through the comprehensive comparison, according to the theoretical analysis of the scattering characteristics of light space, 
combined with the BRDF (Bi-directional Reflection Distribution Function) illumination model, the LED panel lamp is designed and selected as the light source. The service life is long, the lighting of the panel is uniform, and the lamp and the lamp are between. The shadow area is not easy to form. At the same time, considering the characteristics of the surface of the conveyor belt and wood veneer, the panel light on the upper side of the light is placed at 45 degrees.

\subsection{Shading Box}

Because the actual detection environment is different, and the natural light intensity of the outside environment is different in different time periods, which have great influence on the image quality of the industrial camera. In order to ensure that the normal working time of the detection system is uniform and stable, the best quality image is collected by the industrial camera, so a wooden shading box is needed to realize the isolation of the working light and the outside. At the same time, in order to reduce the influence of the reflected light inside the shading box on the camera, the black paint will be rubbed on the inner wall of the box.

\section{System Software Design}

This detection system uses Microsoft Visual Studio 2017 as the development platform, uses C\# as the main development language, and PLC control program uses WPL Soft software to write [9].

\subsection{Algorithm Flow Design}

The system software algorithm flowchart is shown in Figure 3.

(1) Image preprocessing operation. Because the image will be polluted to a certain extent during the acquisition and transmission process, in order to get the accurate image of the surface of the wood veneer, the system adopts a composite filter to denoise it. Then, the absolute value of the gray value of the defect image and the mean gray degree is subtracted and then accumulated. As a result, the image is judged to be a defective image. If it is not, if it is, the image needs to be operated on. After the image judgment, the image of the defective image needs to be sharpened by Laplace, and then a clear defect image is obtained.

(2) Image extraction segmentation and feature extraction. The main work of the defect image extraction and segmentation is divided as follows: using the Histogram of Oriented Gradients (HOG) method, the sample image is segmented. First, a small area of $20 \times 20$ pixels is divided, and the gradient of the main direction is calculated by calculating the gradient. Then the gradient arrow with the strongest directivity is replaced by the original pixel square, and the HOG form of the original sample image is converted to highlight the main features of the image; then the sample library of the trained defect type (the sample library) HOG samples from all defects identified in veneers were compared to extract defect regions.

(3) Design training neural network model. Based on the theoretical algorithm of neural network, the BP neural network classification model is established, and a neural network node is optimized by embedding a large number of measured values into the neural network nodes. Then the design training of the defect convolution neural network model is completed, and then the defects of the wood veneer are distinguished according to the trained model type. 


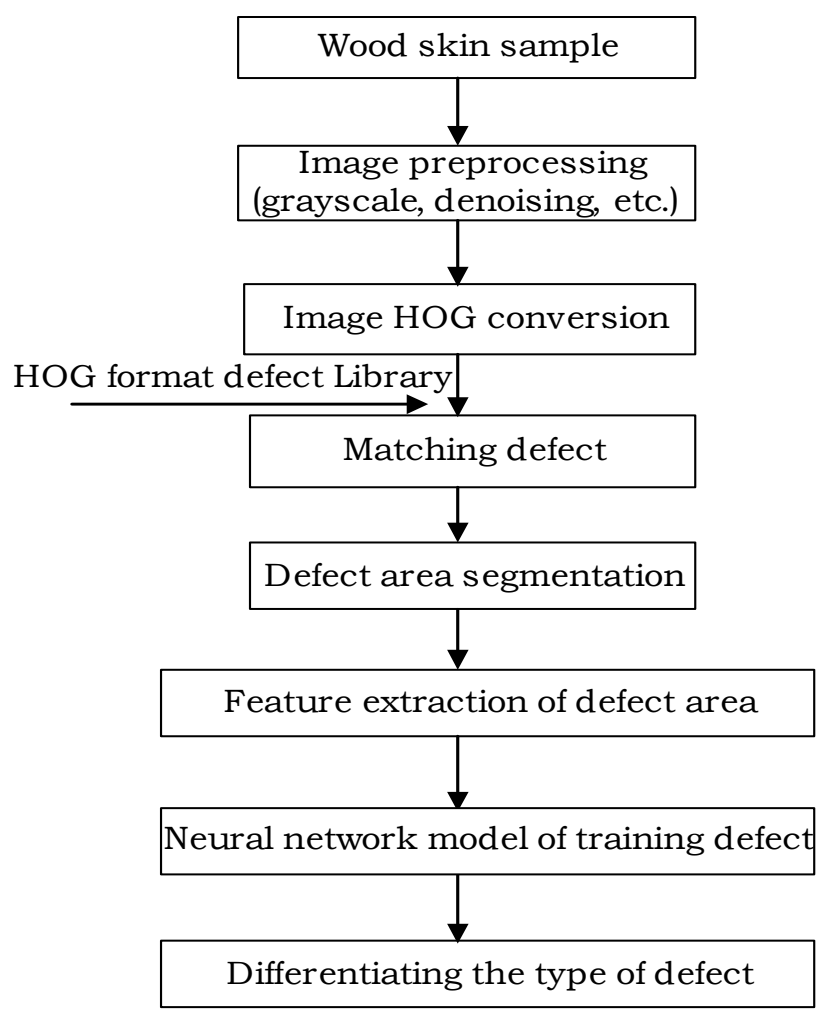

Fig. 3 System software algorithm flow chart.

\subsection{System Interface}

Figure 5 is a human-machine interface diagram of the system design.

(1) Image display area: mainly used to display real time images captured by camera and system software to detect and identify results. The actual detection effect of the system on wood skin detection is shown in Figure 4. The area enclosed by the blue wire frame represents the hole area detected by the software system, the red line frame circles the area that represents the color spot scar area detected by the software system, and the yellow line frame is circled by the yellow wire frame to represent the rough outline shape of the wood skin detected by the software system.

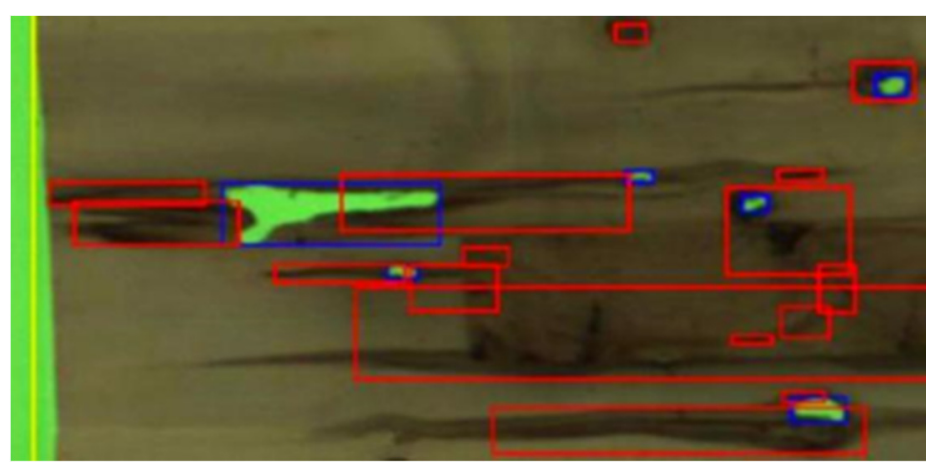

Fig. 4 Local effect map of wood skin defect detection.

(2) Function area: it is mainly used to set and identify the parameters before software acquisition, and then output the relevant identification data. It includes identification parameters, image acquisition, recognition results and visual testing.

(1)Identification parameters: the basic parameters of the defective wood skin are set, including the size of the defects to be detected, the size of the display area, the camera environment parameters and the calibration of the camera.

(2)Image acquisition: setting up camera and collecting pictures.

(3)Identification results: output data after testing wood veneer, including types and quantities of defects. 
(4)Visual testing: the image captured by the camera under non-pipeline conditions can be detected to verify the defect detection effect.

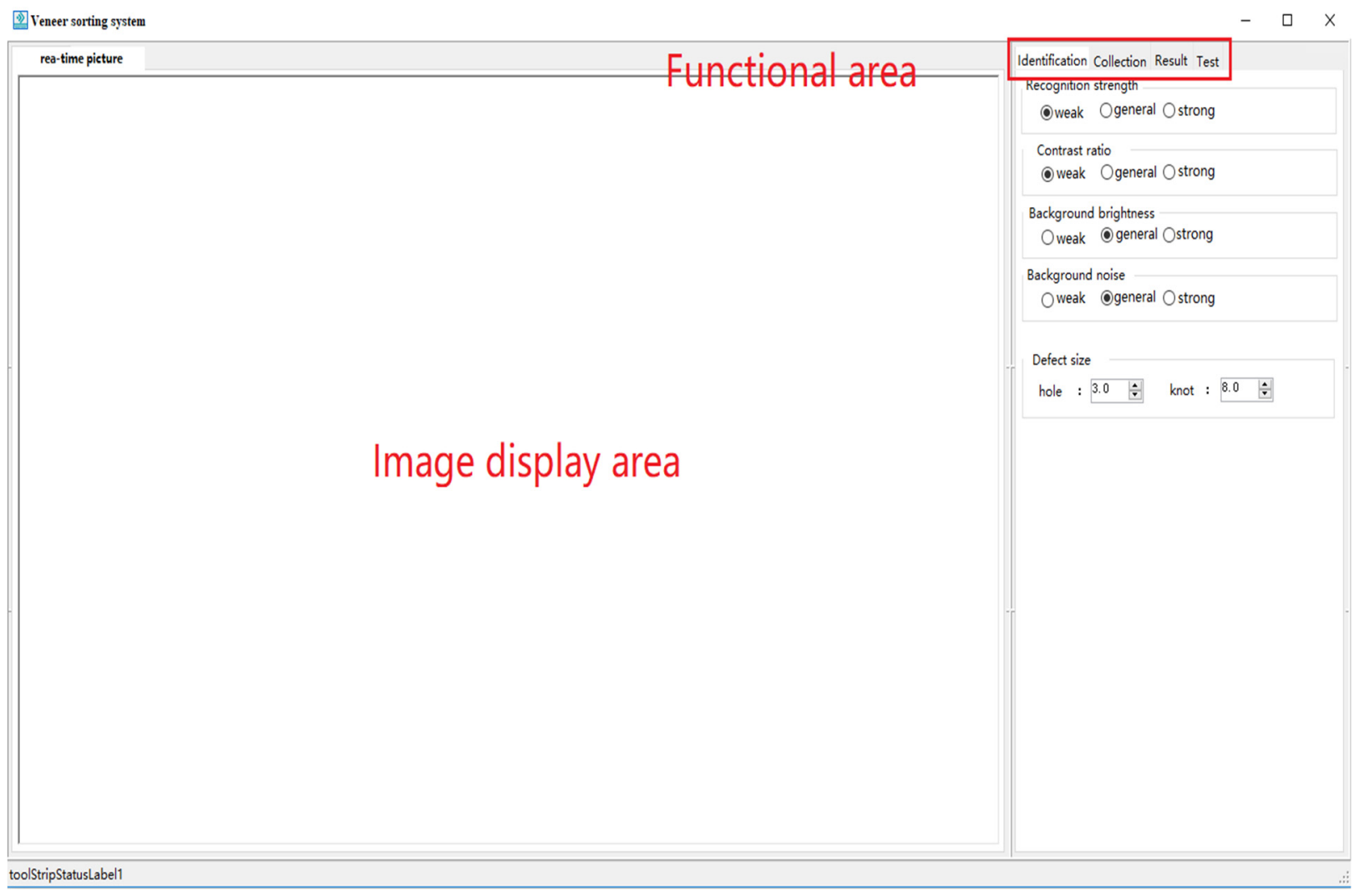

Fig. 5 Man-machine interface diagram

\section{Experiment and Result Analysis}

In order to verify the reliability of the system, we detect and identify wood veneer samples containing four kinds of defects including slipknot, dead knot, crack and hole. The experiment uses the features of HOG, FREAK and SURF and four types of kernel functions of Gaussian Kernel, Polynomial Kernel, Chi-Square Kernel and Histogram intersection kernel to test the four types of defects. The experiment selects 90 defect images as training samples, and also selects 60 defect images as test samples. The computer hardware platform used in the test is: Intel Core i7-7700 CPU (quad core, $3.60 \mathrm{GHz}$ ). The results of the test are shown in table 1-4.

Table 1. The results of Gauss kernel function and three feature recognition defects.

\begin{tabular}{cccccc}
\hline Feature & Dead knot & Slipknot & Crack & Hole & Average rate \\
\hline HOG & $95.00 \%$ & $88.33 \%$ & $90.00 \%$ & $88.33 \%$ & $90.42 \%$ \\
FREAK & $95.00 \%$ & $88.33 \%$ & $88.33 \%$ & $86.67 \%$ & $89.58 \%$ \\
SURF & $91.67 \%$ & $90.00 \%$ & $86.67 \%$ & $88.33 \%$ & $89.17 \%$ \\
\hline
\end{tabular}

Table 2. The results of Polynomial Kernel function and three feature recognition defects.

\begin{tabular}{cccccc}
\hline Feature & Dead knot & Slipknot & Crack & Hole & Average rate \\
\hline HOG & $90.00 \%$ & $88.33 \%$ & $90.00 \%$ & $88.33 \%$ & $89.17 \%$ \\
FREAK & $90.00 \%$ & $90.00 \%$ & $88.33 \%$ & $90.00 \%$ & $89.58 \%$ \\
SURF & $90.00 \%$ & $91.67 \%$ & $86.67 \%$ & $86.67 \%$ & $88.75 \%$ \\
\hline
\end{tabular}


Table 3. The results of Chi-Square Kernel function and three feature recognition defects.

\begin{tabular}{cccccc}
\hline Feature & Dead knot & Slipknot & Crack & Hole & Average rate \\
\hline HOG & $91.67 \%$ & $91.67 \%$ & $88.33 \%$ & $91.67 \%$ & $90.84 \%$ \\
FREAK & $88.33 \%$ & $88.33 \%$ & $90.00 \%$ & $91.67 \%$ & $89.58 \%$ \\
SURF & $88.33 \%$ & $88.33 \%$ & $88.33 \%$ & $91.67 \%$ & $89.17 \%$ \\
\hline
\end{tabular}

Table 4. The results of Histogram intersection kernel function and three feature recognition defects.

\begin{tabular}{cccccc}
\hline Feature & Dead knot & Slipknot & Crack & Hole & Average rate \\
\hline HOG & $90.00 \%$ & $90.00 \%$ & $90.00 \%$ & $90.00 \%$ & $90.00 \%$ \\
FREAK & $88.33 \%$ & $88.33 \%$ & $88.33 \%$ & $91.67 \%$ & $89.17 \%$ \\
SURF & $91.67 \%$ & $90.00 \%$ & $88.33 \%$ & $86.67 \%$ & $89.17 \%$ \\
\hline
\end{tabular}

\section{Conclusion}

From table 1-4, we can see that comparing the three characteristics of HOG, FREAK and SURF and four types of nuclear function combination experiment results, it is best to detect the defect image by using the HOG feature and the Chi-Square Kernel combination. At the same time, the detection results also show that the system can accurately detect the defects on the surface of the wood skin, thus providing a guarantee for the optimization of the production of the enterprise.

According to the actual production inspection needs of wood veneer detection production line, a system of wood veneer surface defect detection based on machine vision technology is designed. The actual test results show that the wood veneer defect detection system has great advantages in realtime detection and accuracy, which meets the requirements of actual production and has a good application prospect.

\section{References}

[1]. Wenjun Sun, Baodong Cheng, XinSheng Pang. Comparative analysis of international competitiveness of Chinese plywood [J]. Journal of Beijing Forestry University (SOCIAL SCIENCE EDITION), 2014, 13 (4): 67-68. (2000) 51-59.

[2]. Hongjie Chang, Juyun Sun, Yanfang Yue, Guang Yang. Application of machine vision in surface defect detection of sheet metal [J]. Mechanical design and manufacturing, 2017 (8): 144-146.

[3]. Jianhua Yang, Wei Zhang, Li Li. Design of surface defect detection system for sawn timber based on machine vision [J]. Forest products industry, 2013 (1): 21-22.

[4]. Chongxin Ren, Jianhua Bi, Lin Xie, Junfeng Jun. Image segmentation method is used for quantitative detection of wood surface defects, [J]. Liquid crystal display and 2014, 29 (5): 786787.

[5]. SAHAMEH S, SAEID M. Potential application of machine vision to honey characterization [J]. Trends in Food Science \& Technology, 2013, 30 (2): 174-177.

[6]. SUN T, TSENG C, CHEN Minsheng. Electric contracts inspection using machine vision [J]. Image and Vision Computing, 2010, 28 (6): 890-901.

[7]. Liu Y., Yu F.H. Automatic Inspection System of Surface Defects on Optical IR-CUT Filter Based on Machine Vision [J]. Optics and Lasers in Engineering, 2014, 55: 243-257.

[8]. Kurtulmus F., Ulu T. C. Detection of Dead Entomopathogenic Nematodes in Microscope Images Using Computer Vision [J]. Biosystems Engineering, 2014, 118: 29-38.

[9]. Zhongda Telecom Limited by Share Ltd. DVP PLC Application Technology Manual [K], 2012. 